\title{
Camera Color Correction Using Normalized Signal Polynomial Regression
}

\author{
Yu-Che Wen ${ }^{1}$, Senfar Wen ${ }^{1}$, Yu-Hsiung Lin ${ }^{2}$ \\ ${ }^{1}$ Department of Photonics Engineering, Yuan Ze University \\ Chung-Li, Taiwan \\ s1025620@mail.yzu.edu.tw; swen@saturn.yzu.edu.tw \\ ${ }^{2}$ Department of Electrical Engineering, Chung Hua University \\ Hsinchu, Taiwan \\ bear@chu.edu.tw
}

\begin{abstract}
A camera color correction method based on the polynomial regression using normalized signal components is proposed. The computation cost of this method can be significantly reduced in comparison with root-polynomial regression method, while the linear relation between signal vector and tristimulus vector is still kept. Performance tests show that the average color difference and median color difference of this method are about the same as that of root-polynomial regression method; the maximum color difference of this method can be apparently reduced in comparison to root-polynomial regression method.
\end{abstract}

Keywords: camera, device model, color calibration

\section{Introduction}

The spectral responses of a camera are the products of the transmittances of color filters and the spectral response of image sensor, such as CCD or CMOS. The raw signal vector is designated as $\mathbf{S}=(R, G, B)$, in which $R, G$, and $B$ are signal components of red, green, and blue channels, respectively. If the spectral responses of a camera exactly fit the CIE color matching functions (CMFs), the camera signal vector $\mathbf{S}$ is linearly proportional to the tristimulus vector $\mathbf{T}=(X, Y, Z)$ of image color. However, such an ideal camera cannot be easily realized. If CMFs can be represented as the linear combination of camera spectral responses, the relation of raw signal vector and tristimulus vector also can be related with a $3 \times 3$ matrix [1]. The professional camera developed in [1] is implemented with three image sensors and three interference color filters, in which filter transmittances are carefully tailored so that CMFs can be nearly represented as the linear combination of camera spectral responses. On the other hand, a consumer camera is usually implemented with an image sensor and a IR cut filter, in which there is an absorptive color filter array (CFA) on the image sensor [2]. The transmittances of red, green, and blue filters on the absorptive CFA cannot be tailored as desired. As CMFs cannot be represented as the linear combination of spectral responses of the consumer camera, color calibration is essential for accurately recording image color.

Color calibration is to find a mapping from camera signal vector to tristimulus vector. Such a mapping can be called the camera color device model or camera model for simplicity. To obtain an accurate mapping, it requires a set of color patches under an illuminant. Their tristimulus vectors are measured by a spectroradiometer. For an example, X-Rite ColorChecker SG is a target chart comprising 96 color patches so that the calibrated camera captures the image of the chart and signal vectors corresponding to the color patches can be extracted from the captured image. Usually the irradiance on the chart is not uniform. The non-uniformity of irradiance can be compensated by taking the image of a gray card under the same illumination condition. Reference [3] proposed a compensation method without taking the image of gray card.

The signal vectors and tristimulus vectors of color patches can be related by regression [4,5]. The use of linear regression has the advantage of linear relation between signal vector and tristimulus vector but is less accurate in comparison to the use of polynomial regression. The linear relation means that the tristimulus vector corresponding to the signal vector $k \boldsymbol{S}$ is $k \boldsymbol{T}$, in which $k$ is a positive constant depending on irradiance. Unfortunately, the linear relation is not valid for the case using polynomial regression. Reference [6] proposed a method using root-polynomial regression for 
keeping the linear relation. Camera color device models using linear regression, polynomial regression, and rootpolynomial regression are called the linear regression model (LRM), polynomial regression model (PRM), and rootpolynomial regression model (RPRM), respectively.

This paper proposes a calibration method for correcting the camera responses to tristimulus values. This method is based on the polynomial regression using normalized signal components for reducing computation cost while the linear relation between signal vector and tristimulus vector is still kept. This method is called the normalized signal regression model (NSRM). The performance of NSRM is evaluated in comparison to that of LRM, PRM, and RPRM.

\section{Camera Color Device Models}

\subsection{Background}

A device model can be written as

$$
\mathrm{q}=\mathrm{Mp}
$$

In (1), $\mathbf{q}$ and $\mathbf{p}$ are column matrixes comprising desired output values and camera responses, respectively. For LRM, PRM, and RPRM, the matrix $\mathbf{q}=(X, Y, Z)^{\mathrm{T}}$. The terms in the matrix $\mathbf{p}$ depends on regression model, e.g.,

$$
\mathbf{p}=(R, G, B)^{T} \text { for LRM, }
$$

$\mathbf{p}=\left(R, G, B, R^{2}, G^{2}, B^{2}, R G, G B, B R, R^{3}, G^{3}, B^{3}, R G^{2}, R B^{2}, G B^{2}, G R^{2}, B R^{2}, B G^{2}, R G B\right)^{T}$ for the third-order PRM,

$$
\mathbf{p}=\left(R, G, B, \sqrt{R G}, \sqrt{G B}, \sqrt{B R}, \sqrt[3]{R G^{2}}, \sqrt[3]{R B^{2}}, \sqrt[3]{G B^{2}}, \sqrt[3]{G R^{2}}, \sqrt[3]{B G^{2}}, \sqrt[3]{B R^{2}}, \sqrt[3]{R G B}\right)^{T} \text { for the third-order RPRM }
$$

For PRM and RPRM, the third-order regression is taken as an example because it is the optimal order for the cases shown in this article. The third order is also the optimal order for [6]. The number of terms in p is denoted as L. From (3) and (4), $\mathrm{L}=19$ and 13 for the third order PRM and RPRM, respectively.

$\mathrm{M}$ is a matrix relating $\mathrm{q}$ and $\mathrm{p}$, which can be found by regression in least-square-fitting sense. Given a set of $\mathrm{N}$ known $\mathrm{XYZs}$ for a reflectance target chart and the corresponding camera responses, we may construct a $3 \times \mathrm{N}$ matrix $\mathrm{Q}$ from $\mathrm{N}$ column matrixes q and a NxL matrix P from N column matrixes p. Using the Moore-Penrose inverse, we have

$$
\mathbf{M}=\mathbf{Q} \boldsymbol{P}^{T}\left(\boldsymbol{P} \boldsymbol{P}^{T}\right)^{-1}
$$

\subsection{Normalized Signal Regression Model (NSRM)}

The proposed NSRM takes the following normalizations for camera responses and tristimulus values. The normalized responses are

The normalized responses are

$$
\begin{aligned}
& r=\frac{R}{R+G+B} \\
& g=\frac{G}{R+G+B}
\end{aligned}
$$

$$
\begin{aligned}
& \alpha=\frac{X}{R+G+B} \\
& \beta=\frac{Y}{R+G+B} \\
& \gamma=\frac{Z}{R+G+B}
\end{aligned}
$$


The normalized parameters $r, g, \alpha, \beta$, and $\gamma$ are independent of irradiance because all the values of $R, G, B, X, Y$, and $Z$ are linearly proportional to irradiance. Equation (1) is also taken for transforming $(r, g)$ to $(\alpha, \beta, \gamma)$, in which $\mathbf{q}=(\alpha, \beta, \gamma)^{\mathrm{T}}$ and

$$
\mathbf{p}=\left(1, r, g, r^{2}, g^{2}, r g, r^{3}, g^{3}, r g^{2}, g r^{2}\right)^{T}
$$

for the third order NSRM. The third order is also the optimal order. The number of terms in $\mathbf{p}$ shown in $(8)$ is $L=10$. The matrix $\mathbf{M}$ in (1) also can be found with (5) for NSRM.

Given a signal vector $\mathbf{S}=(R, G, B)$ and the matrix $\mathbf{M}$, the corresponding tristimulus values can be calculated with the following steps

Step 1: calculate the values of $r$ and $g$ from (6a) and (6b).

Step 2: calculate the values of $\alpha, \beta$, and $\gamma$ from (1), in which the column matrix $\mathbf{p}$ is given by (8).

Step 3: $X=\alpha(R+G+B), Y=\beta(R+G+B)$, and $Z=\gamma(R+G+B)$.

The computation cost with NSRM can be significantly reduced in comparison to that of RPRM. Calculating a set of tristimulus values with the NSRM requires 29 additions, 40 multiplications, and 2 divisions. Calculating a set of tristimulus values with the RPRM requires 36 additions, 56 multiplications, 3 square roots, and 7 cubic roots. In addition, the hardware implementations of square root and cubic root in a camera image processing chip are costly.

Table 1: $\Delta E_{00}$ statistics for multiple calibration models, including linear regression model (LRM), polynomial regression model (PRM), root-polynomial regression model (RPRM), and normalized signal regression model (NSRM). CIE D65 and two lamps of correlated color temperature $6500 \mathrm{~K}$ are respectively taken as illuminants, in which the lamps are compact fluorescence lamp (CFL) and LED lamp.

\begin{tabular}{|c|c|c|c|c|c|c|c|c|c|}
\hline Camera & \multicolumn{3}{|c|}{ D65 } & \multicolumn{3}{c|}{ CFL } & \multicolumn{3}{c|}{ LED Lamp } \\
\cline { 2 - 10 } Model & Average & Median & Maximum & Average & Median & Maximum & Average & Median & Maximum \\
\hline LRM & 1.33 & 1.09 & 4.49 & 0.93 & 0.74 & 3.91 & 1.15 & 0.98 & 3.99 \\
\hline PRM & 0.96 & 0.79 & 4.03 & 0.69 & 0.52 & 4.30 & 0.81 & 0.72 & 3.14 \\
\hline RPRM & 0.77 & 0.60 & 3.36 & 0.58 & 0.44 & 3.38 & 0.66 & 0.51 & 3.08 \\
\hline NSRM & 0.83 & 0.65 & 2.94 & 0.65 & 0.48 & 2.74 & 0.72 & 0.58 & 2.55 \\
\hline
\end{tabular}

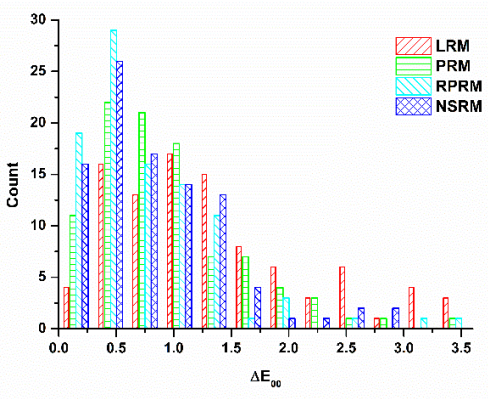

(a)

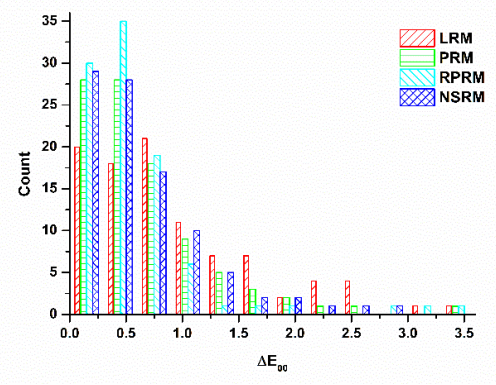

(b)

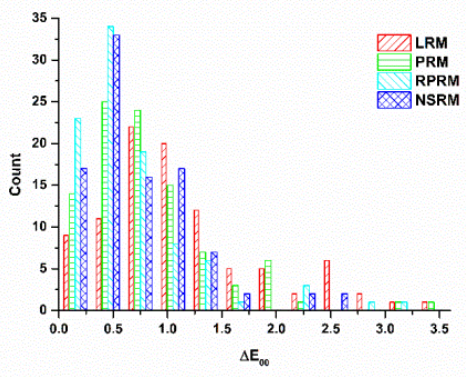

(c)

Fig. 1: $\Delta E_{00}$ histograms for multiple calibration models under (a) illuminant D65, (b) CFL, and (c) LED lamp shown in Table 1. 


\section{Results}

Synthetic data simulation is used to test the performances of LRM, PRM, RPRM, and NSRM so that the uniform irradiance can be assumed. Nikon D5100 is taken as the test camera, in which its spectral responses were shown in [7]. XRite ColorChecker SG was taken as the target chart, in which the spectral reflectance of 96 color patches were measured with Photo Research PR-655 spectrophotometer. Three light sources were respectively taken as the illuminants, which are CIE D65, Philips compact fluorescence lamp (CFL), and Toshiba LED lamp. The correlated color temperature of CFL and LED lamp are $6500 \mathrm{~K}$. The spectral power densities of the two lamps were also measured with PR-655.

A model was evaluated using the leave-one-out method [6]. The model was built from all but one of the color patches and it was tested on the remaining color patch. The color difference $\triangle \mathrm{E} 00$ (CIEDE2000) between the tristimulus values predicted by the model and the ground-truth tristimulus values for the remaining color patch was calculated. The groundtruth tristimulus values were calculated from the spectral reflectance of the remaining color patch and the spectral power density of illuminant. This procedure was repeated for all 96 color patches. The average color difference, median color difference, and the maximum color difference for all 96 color patches were calculated.

Table 1 shows $\triangle E 00$ statistics for LRM, PRM, RPRM, and NSRM. The cases with the illuminants CIE D65, CFL, and LED lamp are shown. The performance of LRM is the worst as expected. The performances of RPRM and NSRM are better than that of PRM in addition to the linear relation between the signal vector and tristimulus vector. The average color difference and median color difference of NSRM are slightly larger than that of RPRM. However, the increases of the average color difference and median color difference of NSRM are less than 0.07 . On the contrary, the maximum color difference of NSRM is less than that of RPRM. The decreases of the maximum color difference of NSRM are $0.43,0.64$, and 0.52 with illuminants CIE D65, CFL, and LED lamp, respectively, in comparison to RPRM. Figure 1 shows $\triangle E 00$ histograms for the cases shown in Table 1. We can see that the use of NSRM is able to reduce the maximum color difference.

\section{Conclusion}

Normalized signal regression model is proposed for correcting the camera responses to tristimulus values. The computation cost can be significantly reduced while the linear relation between the signal vector and tristimulus vector is still kept. Performance tests show that the average color difference and median color difference of normalized signal regression model are about the same as that of root-polynomial regression model; the maximum color difference of normalized signal regression model can be apparently reduced in comparison to root-polynomial regression model.

\section{Acknowledgements}

The work was supported in part by Ministry of Science and Technology, R. O. C., under Contract No. MOST105-2221-E-155-026.

\section{References}

[1] K. Masaoka, Y. Nishida, T. Soeno, T. Yamashita, M. Sugawara, and A. Saita, "Designing Camera Spectral Sensitivities for UHDTV," SMPTE Mot. Image Jour., vol. 123, pp. 26-32, 2014.

[2] G. C. Holst and T. S. Lomheim, CMOS/CCD Sensors and Camera Systems, 2nd Ed. Bellingham WA: SPIE Press, pp. 143-149, 2011.

[3] G. D. Finlayson, M. M. Darrodi, and M. Mackiewicz, "The alternating least squares technique for nonuniform intensity color correction," Color Res. Appl., vol. 40, no. 3, pp. 232-242, 2015.

[4] H. R. Kang, Computational Color Technology. Bellingham WA: SPIE Press, pp. 135-150, 2006.

[5] R. S. Berns, Principles of Color Technology, 3rd Ed., New York: John Wiley \& Sons, pp. 215-223, 2000.

[6] G. D. Finlayson, M. Mackiewicz, and A. Hurlbert, "Color correction using root-polynomial regression," IEEE Trans. Image Proc., vol. 24, pp. 1460-1470, 2015.

[7] M. M. Darrodi, G. Finlayson, T. Goodman, and M. Mackiewicz, "Reference data set for camera spectral sensitivity estimation," J. Opt. Soc. Am. A, vol. 32, pp. 381-391, 2015. 